\title{
Adaptive Exercises Generation using an Automated Evaluation and a Domain Ontology: The ODALA+ Approach
}

doi:10.3991/ijet.v6i2.1562

\author{
Farida Bouarab-Dahmani ${ }^{1}$, Malik Si-Mohammed ${ }^{1}$, Catherine Comparot ${ }^{2}$, Pierre-Jean Charrel $^{2}$ \\ ${ }^{1}$ Mouloud Mammeri University, Tizi-Ouzou, Algeria \\ ${ }^{2}$ IRIT-UTM, IC3, Toulouse 2 University, Toulouse, France
}

\begin{abstract}
Generating adapted learning contents is one of the most important activity that can give the best progression of a learner. This is why research on personalization of learning is growing to get learning environments able to adapt the learning activities and contents to the learner's profile. This last contains information about knowledge, skills, behavior, of the learner, at a given time of the learning process and for given domains. This information, to be effective, must be collected directly from an evaluation module that the learning system will systematically integrate in particular with the learning by doing mode.
\end{abstract}

After the satisfactory results of our research on teaching domain modeling and automated evaluation of learner's in the case of learning by doing exercises, we propose in this paper the ODALA+ approach which is an extension of the already proposed approach called ODALA (Ontology Driven Auto-evaluation Learning Approach) by adding two steps for learner's profile calculation using the evaluation results and for generating adapted exercises.

Index Terms-Exercises generation, Learner's evaluation, Learner modelling, Learner's profile calculation.

\section{INTRODUCTION}

Before the advent of ICT (Information and Communication Technology), two major periods of research can be distinguished in the history of the use of computer science for an educational/training purpose: the classic CAL (Computer Aided Learning) period where first attempts at ad hoc assistance to the learner were given through tutorials and the period of ICAI (Intelligent Computer-Assisted Instruction) where techniques of artificial intelligence were introduced in the purpose of achieving learning environments with more "intelligence" essentially by taking into account the cognitive abilities of the learner, the domain and pedagogical knowledge.

To guide and support learners in their learning activity, a profile-based adaptation is necessary. A profile is an instance in a given time of the learner's model and is related to the domains concerned by the learning. The goal is to reproduce the behaviour of an experienced examiner who takes decisions about the most suited questions to propose the learner so that his/her skill level will be improved. Instead, the examiner should attempt to adjust the level of difficulty of questions using prior information concerning the learner's progression in the concerned domain, collected in the learner's model.
Our previous work about ontological teaching domain modelling [1][2] and automated evaluation of learners' knowledge [3][4] proposed the ODALA approach. This last, over the learning sessions outcomes assessment of the learner in terms of marks and indicators that allow a quantitative estimate of the learner's state of knowledge even when solving open questions. The profiles calculus discussed in this paper uses this estimate of the learner's progression in combination with adequate educational settings to get information used by the exercises generation process. As a result, the exercises proposed, after that, are the best indicated for the learner.

The adaptive generation process proposed in this paper consists on choosing a generation strategy which is a conditional sequence of generation scenarios. Each scenario is itself a conditional sequence of generation primitives defined as reusable procedures or functions that execute, in a learning system, the different steps to get automatically exercises adapted to the learner's profile. In addition, elimination primitives can be activated to adjust the exercises number according to what is indicated by the pedagogical supervisor.

After a brief presentation of learner's modelling and the problematic to get adaptive learning systems, our proposals for profile calculation and exercises generation are explained at the third point by introducing OADLA+ approach. Then, the evaluation of our approach through the integration of a module for exercises generation to the WebSiela $^{1}$ system already developed in our precedent works for algorithmic teaching is discussed. We conclude, at the last point, on the contribution of our results and our prospects for immediate research.

\section{LEARner Modelling, PROFile CALCUlation AND ADAPTED CONTENT GENERATION}

The main parts of a typical architecture of computer learning by doing system where we situate our research are presented by Fig. 1. Knowledge about different teaching domains, different pedagogical approaches and about learners are represented in the different bases or modules of the system.

Learner modelling is defined as the system's belief about a learner's state of knowledge [5]. This is one of the most important design activities that determine the "intelligence" level of any computer based learning. This model

${ }^{1}$ Système Intelligent pour l'Apprentissage d'un Langage Algorithmique via le Web 


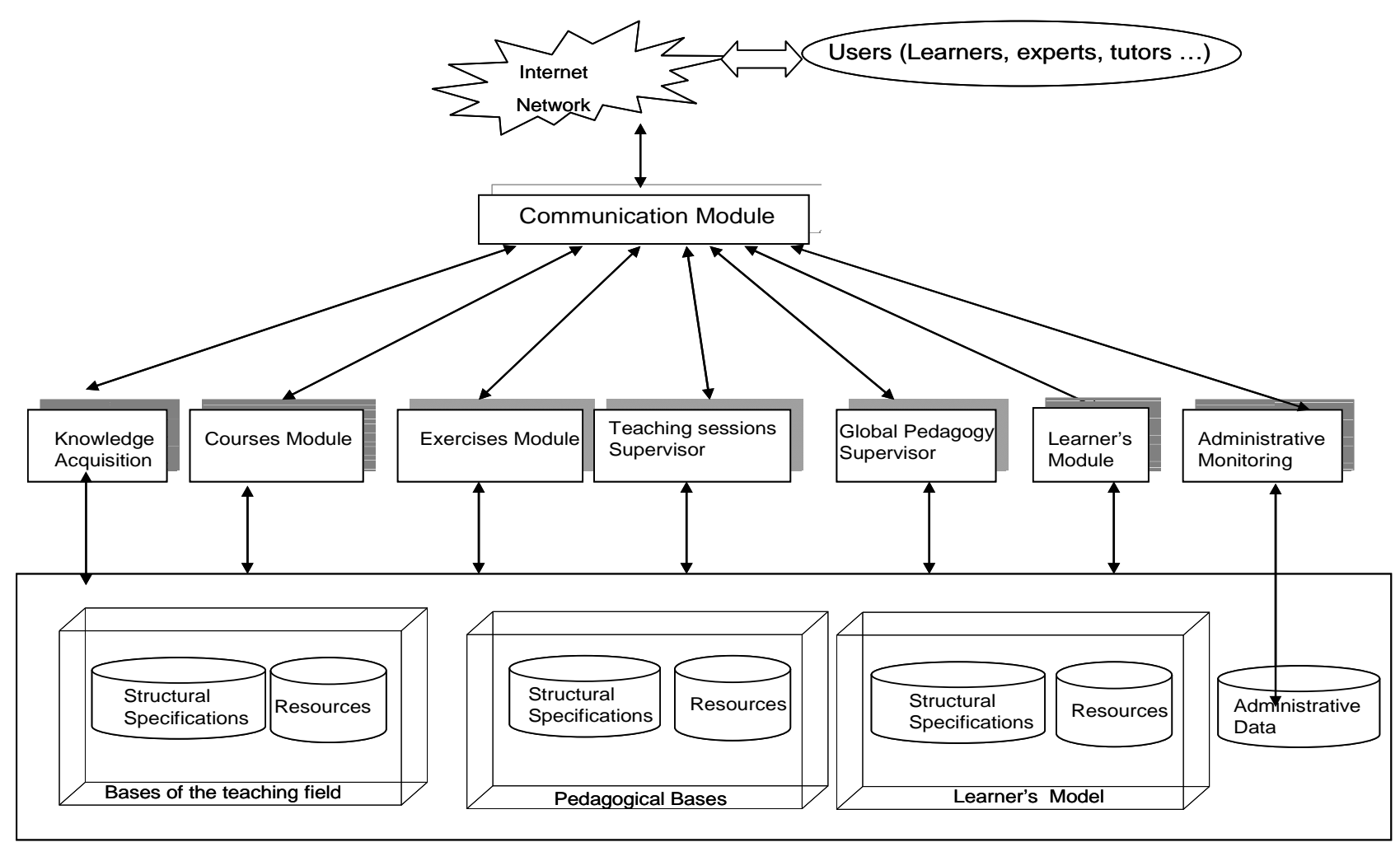

Figure 1. The main components of a computer e-learning by doing system

also affects the system's ability to give individualized learning paths and contents to its users.

The learner profile is to differentiate from the learner model. Indeed, the learner model is, like the models handled in Artificial Intelligence (AI), an accurate reflection of the knowledge of a learner that can be used instantiated for different learning sessions or periods and for different domains. A learner's model parameters must be domain and learning session independent. On the other side, a profile, on a given time, and for a given teaching domain is an instance of this model.

Different type of models have been proposed for the learner such as: the historical model where the system stores historical information about the learner's activities, the buggy model [6] which stores errors in addition to the learner's knowledge and the overlay model [7] that is based on the assumption that knowledge in the learner model may simply be a subset of those of an expert so a subset of the domain model. We have also the multidimensional model [8][9] for a user in general adapted for learning systems. This one represents user profile as several types of information: interests, purposes, historical information ... Each type of information is a dimension in the multidimensional model. This type of user profile is used in most applications and particularly adaptive educational applications. In the dedicated environment for adaptive hypermedia Scarce (Semantic \& Adaptive Composition Environment and Retrieval [10] for example, the user is represented by five dimensions: personal, preferences, knowledge, historical and sessions. In the educational field, several standards of learner representation following the multidimensional model exist and are used for personalization in adaptive educational systems. The most known ones are PAPI (Public And Private Information) ${ }^{2}$ developed by the IEEE and created for communication in collaborative systems and the user model of IMS (IMS LIP $^{3}$ : IMS Learner Information Packaging).

The initialization of a learner model is also problematic for some works about adaptive learning systems. Two types of initialization are proposed: explicit one by reset to empty the learner model or by using predefined stereotypes and implicit one where the profile is constructed from data on learner interactions with the system. We think that with a good evaluation system capable of prognostic assessment, this problem will quickly be solved. At this stage of our work on the calculation of profiles, we are interested in an explicit initialization using a prognostic assessment based on ODALA approach.

We also see through studies on e-learning platforms (such as ORAVEP [11]) to get informed about the state of the art concerning the individualization of learning using profile calculus. We note that the concerns of platforms publishers are often technical and consist to seek solutions to incorporate as many standards as possible of materials and documents formats by giving tools for creating and managing these documents. Educational issues are often placed in the background. If these platforms can democratize undoubtedly courses to mass at relatively low costs, however they are based on a traditional model of teaching and educational support. Learning activities are reduced most often to visit a course and to answer simple questions. Carry out activities more engaging, based on active learning (learning by doing), such as exercises that require the manipulation of the semantics of the subject taught, is

\footnotetext{
${ }^{2}$ http://edutool.com/papi/

3 IMS-LIP (2001), v1.0

http://www.imsglobal.org/profiles/lipbest01.html
} 
not considered. These platforms are rather in a logical transmission of knowledge.

Going from these observations, works have been conducted to provide in general a solution to the problem of guidance and support of distant learners. Three solutions have been proposed: one is to involve the human tutoring itself, the other is to integrate a fully guidance by intelligent tutoring systems. Between these two extremes, there may be of course a third option to share the roles of the human tutor and the automated guidance.

In addition of the necessary guidance, the learner requires that his/her vision is taken into account, which implies taking into account his/her profile (knowledge, effort, preferences, weaknesses, shortcomings, skills, ways of learning, method of progress ...). Therefore, in a learning environment, the concern is to present educational content that best suits the learner by using this profile. An adaptive learning environment can transmit a personalized knowledge to the learner. The use of learners' profiles is one of the ways to adapt learning to the specific characteristics of learners. It is also a way to help the teachers and/or tutors in their task of monitoring [12]. However, systems that manage and operate learner profiles are rare [13]. To do so, the mechanism of adaptability requires a collection of updated information on the learning as far as running sessions to establish individualized learning paths. We recommend that automated assessment based on good teaching domains models is very important to achieving this mechanism. This is the main reason that makes our works on the profile calculation and adaptation of content after those carried out jointly on the ontological modelling of domains and the automated evaluation of learners' knowledge.

The next point of this paper presents our proposals concerning exercises generation adapted to the learner's profile which is calculated using evaluation results as given by a system developed with the ODALA approach.

\section{THE ODALA+ APPROACH}

The ODALA approach proposes a methodology and techniques for developing an evaluation system based on teaching domain ontology Onto-TDM ${ }^{4}$. This approach provides designers of automated learning by doing systems a way of addressing the development problem of the evaluation system from two view points: the developer's and the user's [14].

The main concepts used in Onto-TDM for teaching domain representation in the case of learning by doing are [1]: the evaluation units (a generic class concept where we find exercises, questions, ...), the Notions which are structural components of the teaching domain, including in particular the knowledge items (KI) that are designated as the granular or smallest notions of the domain, and the potential errors of the learner when solving the domain's evaluation units. We also defined taxonomy for errors classification to facilitate their management in the evaluation system and the teaching domain model [2] where different types are proposed: form errors, semantic errors, specific semantic errors, syntactic errors ...

For an automated evaluation system development, the ODALA approach proposes five stages [3]: knowledge base construction by Onto-TDM instantiation, form ana-

\footnotetext{
${ }^{4}$ Ontological Teaching Domain Model
}

lyser construction, semantic analyser construction, marking module integration and learner's model update module

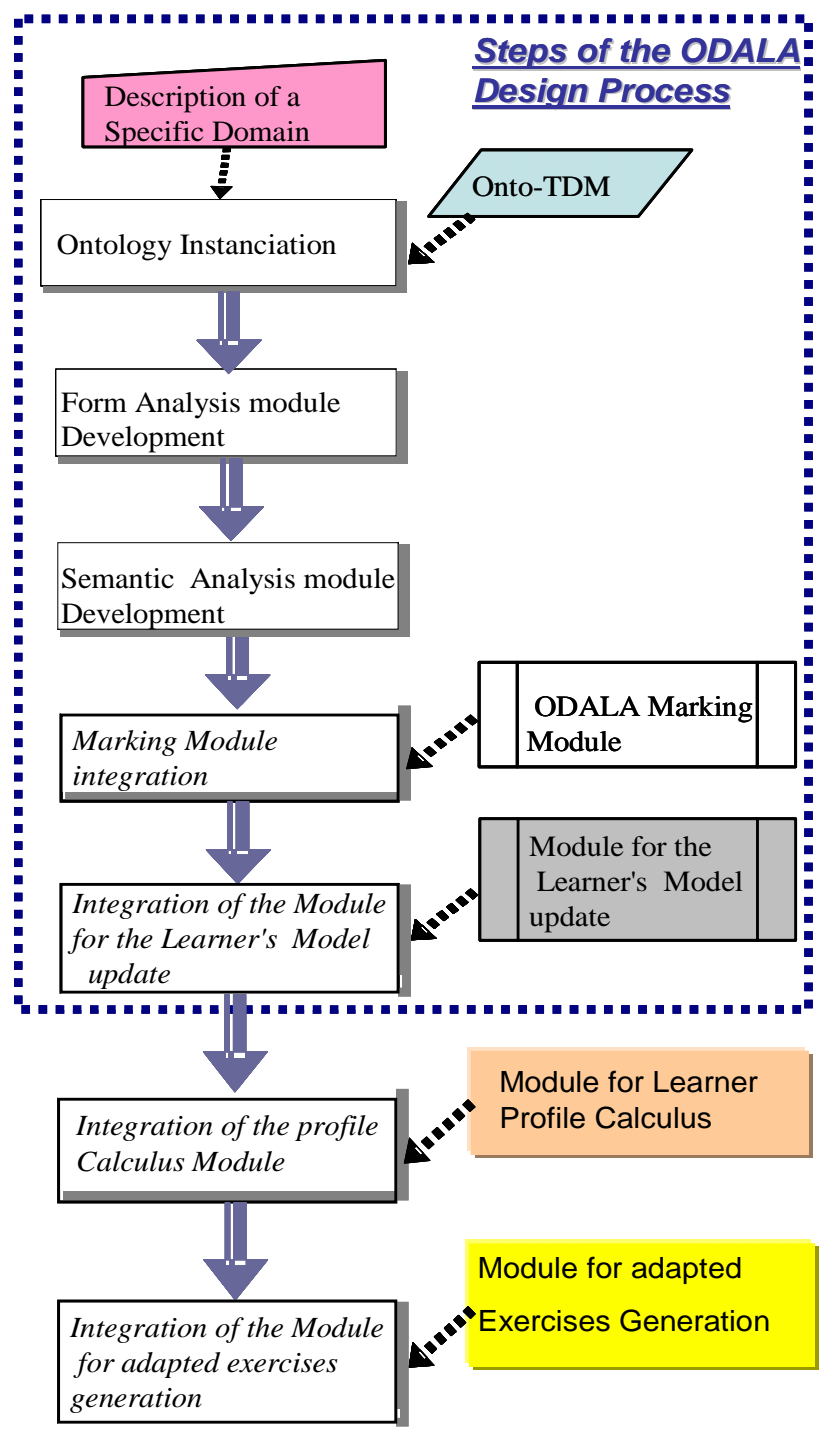

Figure 2. The main steps for developing an evaluation and adapted exercises generation with the ODALA+ approach

integration. Marking and learner's model update modules are proposed reusable by ODALA.

ODALA+ process for designer, as shown by Fig. 2 adds two other steps to ODALA related to learner's profile calculation and automated generation of appropriate exercises.

The following sections of this paragraph illustrate our proposals concerning the different aspects of our approach for learners' profiles calculation and for appropriate exercises generation based on domain modelling and automated evaluation as given by ODALA approach.

\section{A. Learner Modelling}

The learner model reflects the state of the learner (behaviour, skills, weaknesses, preferences ...). It contains in particular the model of his/her knowledge and skills in a given teaching domain. In addition to the different matrices proposed earlier as part of the ODALA approach for storing the evaluation results [14][15] (e.g.: error matrix, the matrix of understanding, exercise matrix, ...), we pro- 
pose the addition of the two following parts or dimensions to get the learner's model (see Fig. 3):

- The general description of the learner where we mainly consider its identity and its classification according to a given scale level.

- The learner's state of knowledge where it is necessary to synthesize the following data: the knowledge items (KI) acquired, the KI partially acquired, the KI not acquired, the KI not viewed yet, the exercises not done yet, the exercises done not acquired and the exercises done and acquired. The degree of acquisition of a KI or an exercise is measured relatively to pedagogical constraints already defined by the pedagogical expert of the learning system. These constraints are presented in the next point.

Thus, compared to the typology of learner's models given in the second paragraph, our approach combines the perception of the overlay model with the buggy model (the matrix of errors is integrated to the Evaluation Results dimension) and multidimensional, with a concentration on the skills dimension.

\section{B. Profile Calculation}

The profile calculation, in the context of our research, is to define at a given time of a learning session or of the learning path, the different values respectively for the parameters of the knowledge dimension described in the ODALA+ learner's model (as given by Fig. 3). We will get then the profile related to a given learner and a given domain as a set of vectors containing respectively acquired KI, acquired done exercises, not done exercises ... These vectors are established based on matrices of the evaluation results' dimension.

\section{Generation of Adapted Exercises}

Using the calculated profile for a given learner, the proposed automated exercises generation module chooses a set of adequate exercises in the sense that they are selected, as far as possible, to resolve the learner's weaknesses according to this profile and the pedagogical context defined by a set of generation constraints (GC). These last are among the generation tools given by our approach and introduced in the following point.

1. Generation tools. The main generation tools used to get adapted exercises to the learner's state of knowledge and the current pedagogy are:

- Generation constraints. We have defined three main acquisition thresholds $X, Y$ and $Z$ to decide about: the acquisition of a KI given its mark in the understanding matrix [15] using X and Y and the acquisition of an exercise given its mark in the exercise matrix using $\mathrm{Z}$ as shown by Fig. 4 . We have also the Nmax parameter (which is the exercises number to generate) and two Boolean constraints: $\mathrm{P}$ to indicate whether it is possible to regenerate exercises already resolved and $\mathrm{Q}$ to precise if $\mathrm{P}$ is True, the exercises to choose: the acquired ones or the not acquired.

- Generation strategies. A generation strategy (see Fig. 5) is defined by a conditional sequence of several generation scenarios.

- Generation scenarios. A generation scenario is a conditional sequence of several generation primitives (see Fig. 5).
- Generation Primitives. These are reusable functions called by the exercises generator. We recommend three types of primitives for: profile calculation, exercises selection according to the profile and the GC and for eliminating exercises used when the number of the exercises generated is upper than Nmax.

2. Steps for Adapted exercises generation. In addition to the calculation of profile that can be called from the generator or run automatically after each execution of the evaluation process, we have these two following steps, each performed by a separate module:

- Exercises selection. This step allows the selection of a set of exercises by activating a generation strategy. This last can be chosen according to the learner's profile. After that, the strategy execution calls different scenarios and each scenario, as shown by Fig. 6, uses different primitives for selecting or eliminating exercises. After execution of a given scenario, we can get Nmax exercises, more (even after elimination primitive execution) or less than Nmax. The tests used by the strategy and those of its scenarios will regulate as far as possible to get the most suited exercises and the closest number of exercises to Nmax.

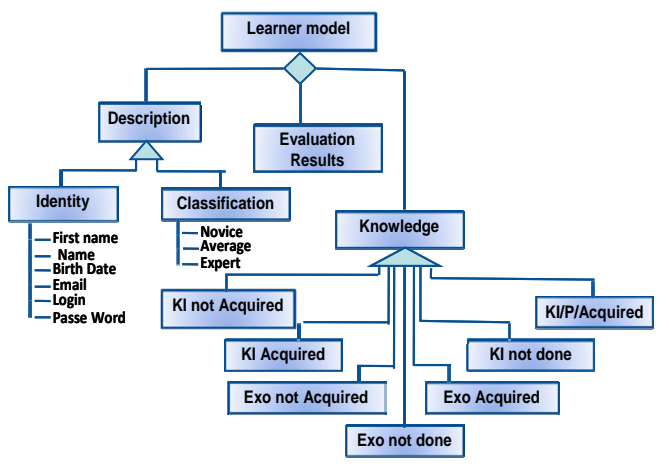

Figure 3. The Learner's model used in ODALA+

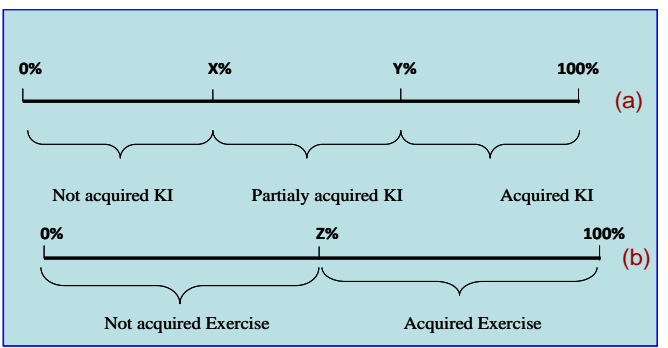

Figure 4. Acquisition thresholds for KI and exercises

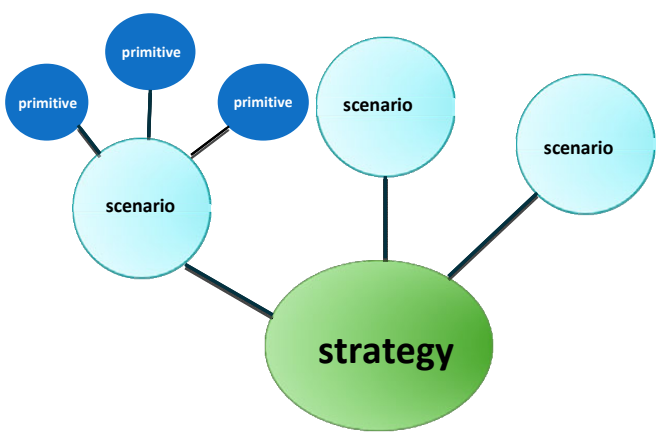

Figure 5. Strategy, scenarios and primitives 
PAPER

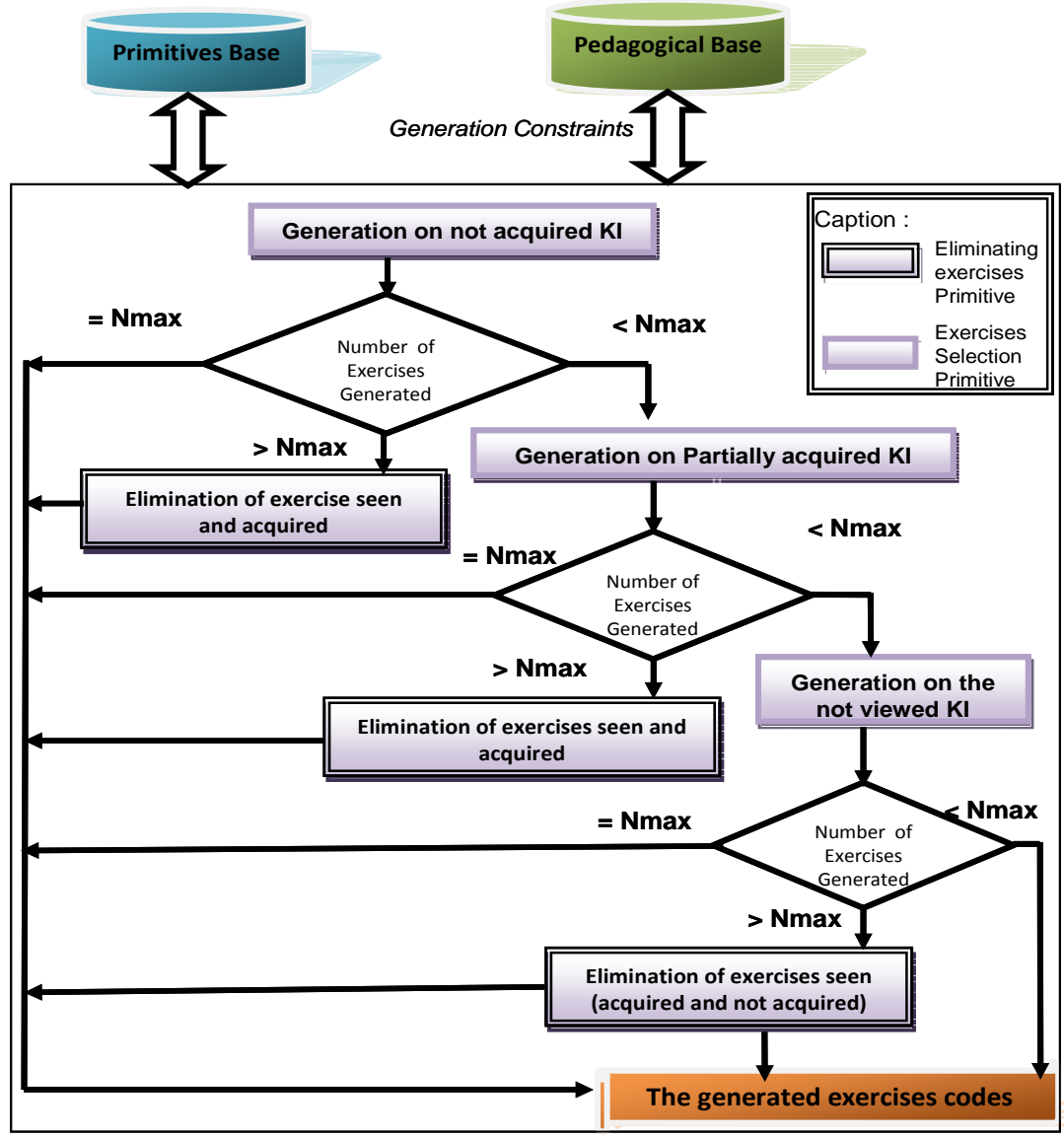

Figure 6. Example of scenario

The example of Fig. 6 shows a case of scenario where the first primitive called selects all the exercises evaluating the KI indicated as not acquired in the calculated learner's profile. After, if we have not yet Nmax exercises, a second primitive will be used to add the exercises evaluating the partially acquired KI, and so on until the end of the scenario.

- Resource recovery. This sub module can retrieve from a resources base (local and/or distant one) the resources (documents, videos ...) corresponding to the resulting list of exercises given by the precedent step. These resources must be pre-selected (by a Web research) or created by an experienced domain teacher. The exercise specification (for example the data properties if the OWL language is used) in the domain ontology indicates the localisation (URL, URI ...) of the different resources connected to the concerned exercise. These localisation properties can be seen as resources annotations commonly used by the Web Semantic community.

The chosen resource by the learner will be displayed in an adequate presentation (see Fig.7).

\section{EVAluation OF OUR PROPOSAls}

The evaluation of ODALA+ is first done by prototype development. Indeed, an automated exercises generation module is integrated, according to ODALA+ steps, to the WebSIELA system already built and tested as part of our works on the ontological teaching domain modelling and automated evaluation of learners when learning by doing algorithmic.

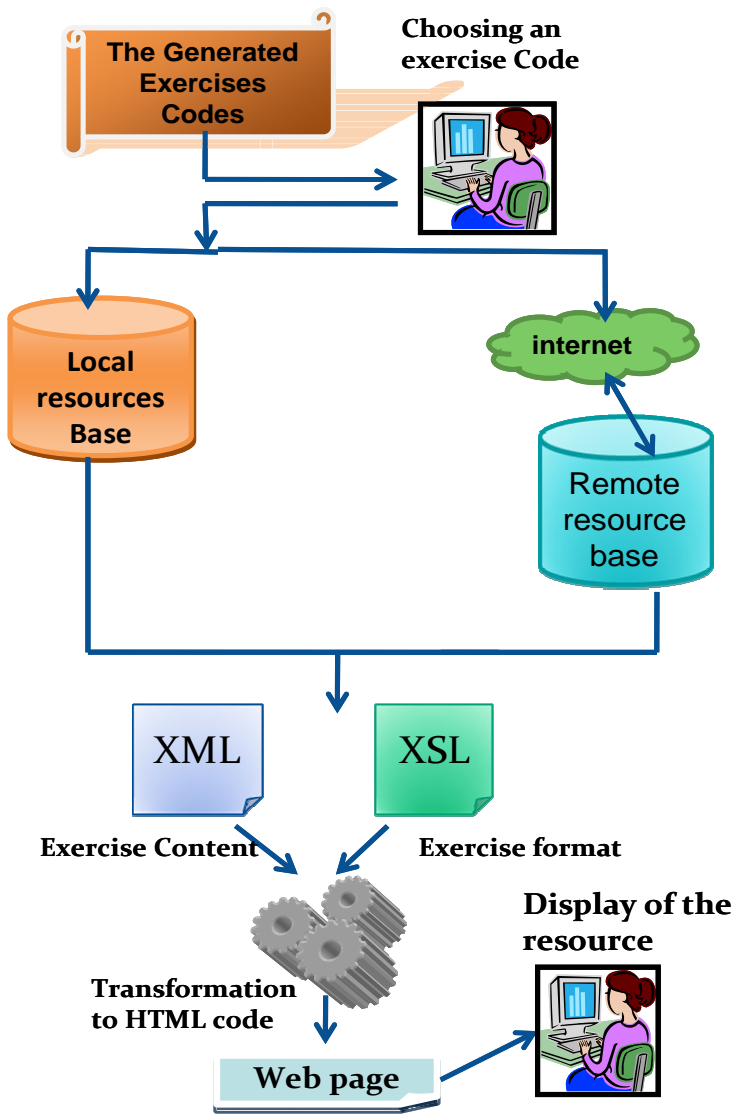

Figure 7. Resource recovery process after adapted exercises generation 
PAPER

A set of tests was then conducted using thirty (30) exercises where different algorithmic notions and knowledge items are evaluated. Ten (10) algorithmic teachers of our university participated in an experiment when each teacher executed at least five learning sessions and controlled, for each session, if the generated exercises are suited to the calculated profile. The learner profile, updated after each evaluation process execution, can be viewed on the author's space (see Fig. 8) and also on the learner's side (as shown by Fig. 9).

We estimated the global satisfaction factor of the ten teachers to $91.3 \%$ (as shown by Table 1). Each factor is calculated using the degree of satisfaction of the teacher after analyzing the list of exercises generated for each executed session. This degree can be: 1 : if satisfied, 0.5 if not yes and not no and 0 if not satisfied. We just add however, that this factor concerns only the exercises generation part. Combined with the global satisfaction factor about the evaluation process estimated to $70 \%$ with the same teachers, the satisfactory factor of the ODALA+ process is approximately $80.65 \%$. The satisfaction factor, obtained for the evaluation process is justified by some facts already explained in our previous papers [4][15].

On the other hand, the experiment allowed us to collect some feedbacks from the teachers regarding the enrich- ment of exercises base, pedagogical strategies and scenarios for exercises generation.

We also plan to integrate these remarks before a second experiment with real students of our university.

\section{CONCLUSION}

We proposed in this paper the part of ODALA+ approach for generating appropriate exercises and learner profile calculation based on a teaching domain modelling and knowledge evaluation results. The first tests with a prototype developed for algorithmic teaching are satisfactory. Our proposals have the advantage of presenting an approach to designing and implementing systems able to generate adapted learning content based on an explicit evaluation in the form of "Glass box" whereas, previously, the evaluation system is either a black box or limited to simple questions management.

ODALA+ gives a continuous way to go from domain modelling to content generation in a coherent and practical method suited for leaning by doing systems. Our immediate research perspective concerns an experimental study about the reusability possibilities of the exercises generation module implemented in WebSIELA.

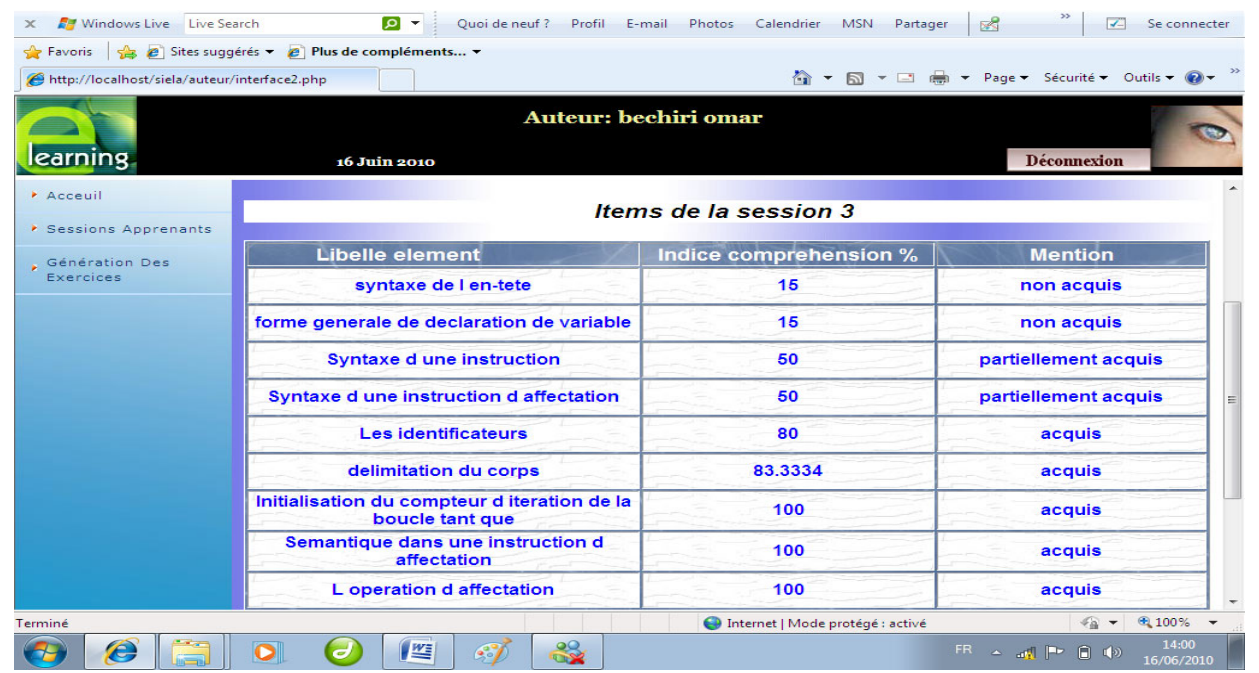

Figure 8. Display of the acquisition KI vector in the side of the author interface with WebSIELA

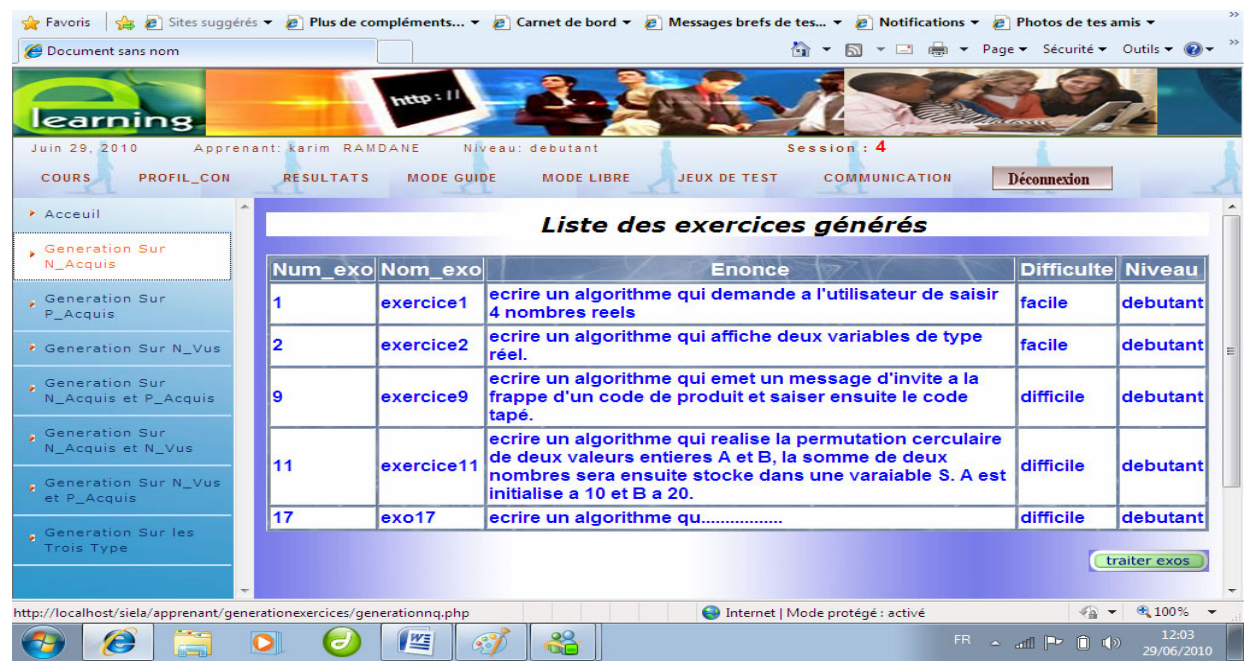

Figure 9. Display of an adapted exercises list resulting from an execution of WebSIELA Generator 
TABLE I.

ESTIMATED FACTORS OF SATISFACTION COLLECTED WHEN TESTING THE WEBSIELA ADAPTED EXERCISES GENERATOR

\begin{tabular}{|c|c|}
\hline Teacher's Number & $\begin{array}{c}\text { Estimated factor of } \\
\text { satisfaction (\%) }\end{array}$ \\
\hline $\mathbf{1}$ & 93 \\
\hline $\mathbf{2}$ & 90 \\
\hline $\mathbf{3}$ & 80 \\
\hline $\mathbf{4}$ & 83 \\
\hline $\mathbf{5}$ & 92 \\
\hline $\mathbf{6}$ & 100 \\
\hline $\mathbf{7}$ & 100 \\
\hline $\mathbf{8}$ & 90 \\
\hline $\mathbf{9}$ & 92 \\
\hline $\mathbf{1 0}$ & 93 \\
\hline Global Estimation & $\mathbf{9 1 , 3} \%$ \\
\hline
\end{tabular}

\section{REFERENCES}

[1] Bouarab-Dahmani F., Si-Mohammed M., Comparot C. \& Charrel P. J. ODALA, an ontological model for automated evaluating of learner knowledge: Application to Teaching of Algorithms, Ecase-2009 (E-education Track) International Conference, Singapore, January 08-10, 2009.

[2] Bouarab-Dahmani F., Si-Mohammed M., Comparot C. \& Charrel P. J. Teaching domain representation and learner's evaluation in a platform for relational databases e-learning, Ecase \& Tech 2010 (E-education Track), International Conference, Macau (Chine), Janvier25-27, 2010.

[3] Bouarab-Dahmani F., Si-Mohammed M., Comparot C. \& Charrel. P. J. Evaluating the learner's state of knowledge when he/she is learning by doing, IADIS International Conference IADIS CELDA 2008, Freiburg, Germany, October 13-15, 2008.

[4] Bouarab-Dahmani F., Si-Mohammed M., Comparot C. \& Charrel P. J. Learners automated evaluation with the ODALA approach, ACM-SAC2009 (Human Computer Interaction Track) International Conference, Honolulu, Hawaii, USA, March 08-12, 2009.

[5] J. Johns, S. Mahadevan, B. Woolf. Estimating student proficiency using an item response theory model. ITS 2006, LNCS 4053, pp 473-480.

[6] Burton R., Brown J-S. Diagnostic Models for procedural bugs in basic mathematical skills, Cognitive Science, Volume 2, pages 155-192, 1978. doi:10.1207/s15516709cog0202_4
[7] VanLehn, K. (1988). Toward a theory of impasse-driven learning. In H. Mandl \& A. Lesgold (Eds.) Learning Issues for Intelligent Tutoring Systems, pp. 19-41. New York, NY: Springer.

[8] Amato G., Staraccia U., « User profile modelling and applications to digital libraries », Proceedings of the 3rd European Conference on Research and advanced technology for digital libraries, 1999, p. 184-187.

[9] Bouzeghoub M., Kostadinov D., « Personnalisation de l'information : Aperçu de l'état de l'art et définition d'un modèle flexible de définition de profils ", Actes de la 2nde Conférence en Recherche d'Information et Applications CORIA, 2005, p. 201218.

[10] S. Garlatti, S. Iksal, et P. Tanguy. Scarce: An adaptive hypermedia environment based on virtual documents and semantic web. In Adaptable and Adaptive Hypermedia System, pages 206-224, 2004.

[11] ORAVEP. Etude comparative technique et pédagogique des plateformes pour la formation à distance », www.oravep.asso.fr, 2000.

[12] GRANDBASTIEN, M., LABAT, J.-M. (2006). Environnements informatiques pour l'apprentissage humain. Traité IC2, série Cognition et traitement de l'information.

[13] S. Jean-Daubias, C. Eyssautier-Bavay, M. Lefevre. Modèles et outils pour rendre possible la réutilisation informatique de profils d'apprenants hétérogènes. Revue Sticef.org, Volume 16, 2009

[14] Bouarab-Dahmani F. Modélisation basée ontologies pour l'apprentissage interactif - Application à l'évaluation des connaissances de l'apprenant. Thèse de DOCTORAT en informatique soutenue à l'UMM de Tizi Ouzou en 2010.

[15] Bouarab-Dahmani F., Si-Mohammed M., Comparot C. \& Charre P. J. Automated Evaluation of Learners with the ODALA Approach: Application to Relational Databases E-learning, International journal of computational intelligence systems, IJCIS, Vol.3, N. 3, (September 2010), 357-369, published by Atlantis press. doi:10.2991/ijcis.2010.3.3.11

\section{AUTHORS}

Dr F. Bouarab-Dahmani is with the Computer science department of Mouloud Mammeri university, Tizi Ouzou, Algeria (e-mail: dahmani_farida@yahoo.fr).

Pr M. Si Mohammed is with the Computer science department of Mouloud Mammeri university of Tizi Ouzou, (e-mail: smalik@assila.net).

Dr. C. Comparot is with the IRIT-UTM-IC3 research team of Toulouse 2 university, France. (e-mail: comparot@univ-tlse2.fr).

Pr. P. J. Charrel is with the IRIT-UTM-IC3 research team of Toulouse 2 university, France. (E-mail: charrel@univ-tlse2.fr).

Received January $31^{\text {st }}$, 2011. Published as resubmitted by the authors May $16^{\text {th }}, 2011$. 